\title{
Effect of serialized messaging on web services performance
}

\begin{abstract}
Message serialization is a format of messaging leveraging Web services to exchange data over the network. Serialized messages are processed at the server and sent as objects over the network to the client to be consumed. While, serialization process minimizes network bandwidth requirement but then incurs overhead at the communicating ends. This research contributes to the study of message exchange using HTTP across communication systems. The research identified the fundamental effect of serializing high-volume messages across network and the sources for the effects at the communication endpoints. The study utilized server - client SOAP Web services to identify the fundamental effect of serialization in the communication endpoints. SOAP messages were exchanged as XML messages over HTTP. Payload sizes (1MB-22MB) for serialized and normal messages were exchanged through the services. The message payload, overhead, and response time were monitored and measured. The overall result indicated that is more beneficial to serialized large payload than smaller one. Generally, the serialization and deserialization cost incurred at individual ends are slightly constant irrespective of the payload size. Also, the serialization and deserialization process is insignificant to the overall transaction as it delay is below $3 \%$ of the total overhead.
\end{abstract}

Keyword: Client-server; Payload; Response time; Overhead 